\title{
Progranulin does not affect motor neuron degeneration in mutant SOD1 mice and rats
}

\author{
Sarah Herdewyn ${ }^{\text {a,b,c }}$, Louis De Muynck ${ }^{\text {a,b }}$, Ludo Van Den Bosch ${ }^{\text {a,b }}$, Wim Robberecht ${ }^{\text {a,b,c }}$, \\ Philip Van Damme ${ }^{\mathrm{a}, \mathrm{b}, \mathrm{c}, *}$ \\ a Laboratory of Neurobiology, Experimental Neurology and Leuven Institute for Neurodegenerative Disorders (LIND) University of Leuven, Leuven, Belgium \\ ${ }^{\mathrm{b}}$ Vesalius Research Center, VIB, Leuven, Belgium \\ ${ }^{\mathrm{c}}$ Department of Neurology, University Hospital Leuven, University of Leuven, Leuven, Belgium
}

\section{A R T I C L E I N F O}

Article history:

Received 12 October 2012

Received in revised form 3 February 2013

Accepted 24 March 2013

\section{Keywords:}

Frontotemporal lobar degeneration

Amyotrophic lateral sclerosis

Motor neuron degeneration

Mutant superoxide dismutase 1

Progranulin

\begin{abstract}
A B S T R A C T
Motor neuron degeneration in amyotrophic lateral sclerosis (ALS) is familial in $10 \%$ of patients, with mutations in SOD1 and C9orf72 being the most frequent cause. There is convincing evidence for overlap between ALS and frontotemporal lobar degeneration at the genetic, pathological, and clinical level. Null mutations in progranulin (PGRN) are a frequent cause of familial frontotemporal lobar degeneration. PGRN exerts neurotrophic properties on motor neurons in vitro and in vivo. We therefore examined whether PGRN could affect disease progression in mutant SOD1 mice and rats, both established models for ALS. Overexpression of PGRN in mice and intracerebroventricular delivery of PGRN in rats did not affect onset or progression of motor neuron degeneration.
\end{abstract}

(c) 2013 Elsevier Inc. All rights reserved.

\section{Introduction}

Amyotrophic lateral sclerosis (ALS) and frontotemporal lobar degeneration (FTLD) are related and sometimes overlapping neurodegenerative disorders, often characterized by ubiquitin- and TAR DNA-binding protein 43 (TDP43) TDP43-positive inclusions in the brain and spinal cord. Some genetic forms of ALS, FTLD, or ALSFTLD have a common cause, such as mutations in C9orf72, or rarely in VCP, TARDBP, CHMP2B, UBQLN2, or the gene encoding PGRN. Mutations in other genes are more specifically associated with 1 phenotype. Mutations in SOD1 are a frequent cause of pure ALS and several established models of mutant SOD1 (mSOD1)-induced motor neuron degeneration exist. Progranulin (PGRN) is a widely expressed pleiotropic growth factor, with neurotrophic properties in vitro and in vivo and it plays a role in neuroinflammation. Loss of function mutations in the gene encoding PGRN are a frequent cause of FTLD, rarely in conjunction with ALS. The resulting haploinsufficiency of PGRN is likely to bring about neurodegeneration in FTLD, but has not been observed in other forms of ALS and/or FTLD. Given the neurotrophic properties of PGRN and the shortage of PGRN involved in 1 form of ALS and/or FTLD, PGRN is a potential

\footnotetext{
* Corresponding author at: Neurology Department, UZ Leuven, Herestraat 49, 3000 Leuven, Belgium. Tel.: +32 16 344280; fax: +32 16344285 .

E-mail address: philip.vandamme@uz.kuleuven.ac.be (P. Van Damme).
}

therapeutic target for ALS and/or FTLD. We therefore examined whether PGRN overexpression or intracerebroventricular (ICV) delivery could affect disease progression in mSOD1 mice and rats, both established models for ALS.

\section{Methods}

Conditional PGRN mice were generated by expression of human PGRN (hPGRN) cDNA preceded by a floxed Neomycine stop cassette in the ROSA26 locus. After crossbreeding these mice to PGK-Cre mice, ubiquitous and constitutive PGRN overexpression mice were obtained. PGRN messenger RNA (mRNA) and protein overexpression was demonstrated using quantitative polymerase chain reaction and enzyme-linked immunosorbent assay. These mice were crossbred to $\mathrm{mSOD} 1^{\mathrm{G} 93 \mathrm{~A}}$ mice. $\mathrm{mSOD} 1^{\mathrm{G} 93 \mathrm{~A}}$ rats were treated with ICV delivery of recombinant PGRN, as previously described. All methods and results are described in detail in the Supplementary data.

\section{Results}

Transgenic mice that overexpress PGRN showed ubiquitous overexpression of PGRN, including overexpression in brain and spinal cord, at the mRNA and protein level. PGRN was expressed in various cell types, as shown using quantitative polymerase chain reaction on cultured (motor and cortical) neurons and glial cells and 
by immunostainings in vivo. Crossbreeding of PGRN overexpression mice to $\mathrm{mSOD} 1^{\mathrm{G} 93 \mathrm{~A}}$ mice did not affect the onset of disease (as determined using the paw grip endurance test and rotarod) or the survival. In addition, quantification of denervated neuromuscular junctions of the gastrocnemius muscle did not show protection. As a second strategy to test the therapeutic potential of PGRN, mSOD1 ${ }^{\mathrm{G} 93 \mathrm{~A}}$ rats were treated with ICV PGRN using miniosmotic pumps connected to an ICV catheter. This resulted in elevated hPGRN levels in cerebrospinal fluid, measured using enzyme-linked immunosorbent assay, but this treatment also failed to show a positive effect on the onset of motor neuron degeneration or survival.

\section{Discussion}

We tested the therapeutic potential of PGRN on motor neuron degeneration induced by mSOD1. Despite the neurotrophic effects of PGRN on cultured motor neurons, overexpression of PGRN or ICV treatment of PGRN did not affect the onset or progression of motor neuron degeneration in $\mathrm{mSOD} 1{ }^{\mathrm{G} 93 \mathrm{~A}}$ rodent models.

These negative results were obtained using 2 complementary strategies: a crossbreeding experiment with overexpression in all cell types, in combination with an ICV delivery experiment, which is directly translatable to patients.

Although negative, these results are in line with what we previously observed in a zebrafish model of mSOD1-induced axonopathy. In zebrafish, hPGRN overexpression alleviated mutant TDP43-induced axonopathy, but had no effect on mSOD1-induced axonopathy. ALS patients with SOD1 mutations do not show ubiquinated TDP43-inclusions, which are present in most of ALS cases. This suggests a divergent disease mechanism for SOD1 and TDP43 and suggests that PGRN might only have beneficial effects in TDP43 proteinopathies.

\section{Disclosure statement}

The authors have no financial or other conflicts of interest.

All experiments on rodents were approved by the local ethical committee of the University of Leuven, Belgium.

\section{Acknowledgements}

The authors thank Sabine Wyns, Ann Bouché, Wendy Scheveneels, and Veerle Crabbé for the excellent technical assistance. We thank the Cell Imaging Core of the University of Leuven for the use of the confocal microscope. This work was supported by grants from SAO-FRMA and ABMM. S.H. holds a PhD fellowship and P.V.D. holds a clinical investigatorship of FWO-Vlaanderen. L.D.M. holds a PhD fellowship of the Agency for Innovation by Science and Technology (IWT). W.R. is supported through the E. von Behring Chair for Neuromuscular and Neurodegenerative Disorders.

\section{Appendix A. Supplementary data}

Supplementary data associated with this article can be found, in the online version, at http://dx.doi.org/10.1016/j.neurobiolaging. 2013.03.027. 